\title{
Identification of potential biomarkers for diagnosis of hepatocellular carcinoma
}

\author{
XING-HUA LIANG ${ }^{*}$, ZHENG-PING FENG ${ }^{*}$, FO-QIU LIU, RONG YAN, \\ LIANG-YU YIN, HAO SHEN and HAI-LIN LU
}

\begin{abstract}
Department of Gastroenterology, The Fourth Affiliated Hospital of Guangzhou Medical University (Zengcheng District People's Hospital of Guangzhou), Guangzhou, Guangdong 511300, P.R. China
\end{abstract}

Received August 23, 2019; Accepted July 7, 2020

DOI: $10.3892 /$ etm.2021.10973

\begin{abstract}
Hepatocellular carcinoma (HCC) has a high mortality rate owing to its complexity. Identification of abnormally expressed genes in HCC tissues compared to those in normal liver tissues is a viable strategy for investigating the mechanisms of HCC tumorigenesis and progression as a means of developing novel treatments. A significant advantage of the Gene Expression Omnibus (GEO) and The Cancer Genome Atlas (TCGA) is that the data therein were collected from different independent researchers and may be integrated, allowing for a more robust data analysis. Accordingly, in the present study, the gene expression profiles for HCC and control samples were downloaded from the GEO and TCGA. Functional enrichment analysis was performed using a Metascape dataset, and a protein-protein interaction (PPI) network was constructed using the Search Tool for the Retrieval of Interacting Genes/proteins (STRING) online database. The prognostic value of mRNA for HCC was assessed using the Kaplan-Meier Plotter, a public online tool. A gene mRNA heatmap and DNA amplification numbers were obtained from cBioPortal. A total of 2,553 upregulated genes were identified. Functional enrichment analysis revealed that these differentially expressed genes (DEGs) were mainly accumulated in metabolism of RNA and the cell cycle. Considering the complexity and heterogeneity of the molecular alterations in $\mathrm{HCC}$, multiple genes for the prognostication of patients with HCC are more reliable than a single gene. Thus, the PPI
\end{abstract}

Correspondence to: Dr Xing-Hua Liang, Department of Gastroenterology, The Fourth Affiliated Hospital of Guangzhou Medical University (Zengcheng District People's Hospital of Guangzhou), 1 Guangming East Road, Guangzhou, Guangdong 511300, P.R. China

E-mail: liangxinghua77@163.com

*Contributed equally

Key words: The Cancer Genome Atlas, Gene Expression Omnibus, hepatocellular carcinoma, early diagnosis, Metascape dataset, protein-protein interaction, Kaplan-Meier Plotter, cBioPortal network and univariate Cox regression analysis were applied to screen candidate genes (small nuclear ribonucleoprotein polypeptide B and B1, nucleoporin 37, Rac GTPase activating protein 1 , kinesin family member $20 \mathrm{~A}$, minichromosome maintenance 10 replication initiation factor, ubiquitin conjugating enzyme E2 C and hyaluronan mediated motility receptor) that are associated with the overall survival and progression-free survival of patients with HCC. In conclusion, the present study identified a set of genes that are associated with overall survival and progression-free survival of patients with HCC, providing valuable information for the prognosis of HCC.

\section{Introduction}

Liver cancer was the sixth most commonly diagnosed cancer type and the fourth leading cause of cancer-associated death worldwide in 2018. Annually, 841,000 new cases of liver cancer are diagnosed and 782,000 deaths are recorded (1). In China, liver cancer is the most commonly diagnosed cancer and the leading cause of cancer-associated death in males below the age of 60 years (2).

Hepatocellular carcinoma (HCC) accounts for $75-85 \%$ of primary liver cancer cases. Despite enormous progress in medical technologies such as surgical resection, liver transplantation, radiation and chemotherapy in recent decades, the 5-year overall survival rate for HCC remains $<30 \%$ (3). Therefore, detailed mechanistic information on the tumorigenesis and progression HCC is increasingly required in order to develop more effective therapeutic strategies.

The identification of abnormally expressed genes between normal liver tissues and HCC tissues is a viable strategy for investigating the mechanisms of HCC tumorigenesis and progression. Furthermore, such differentially expressed genes (DEGs) may serve as prognostic markers for HCC. The National Center for Biotechnology Information (NCBI) Gene Expression Omnibus (GEO, http://www.ncbi.nlm. gov/geo/) is a free public repository for high-throughput gene expression data and offers submission, storage and retrieval of microarray, next-generation sequencing and other forms of functional genomic datasets (4-6). The Cancer Genome Atlas (TCGA; http://cancergenome.nih.gov/) provides both clinical and molecular data on $>11,000$ samples across 33 different tumor types (7). A particular advantage of the 
GEO and TCGA is that the data therein are collected from different independent researchers and may be integrated and applied, allowing for highly robust analyses.

The most fundamental characteristic of cancer cells is their sustained continuous proliferation, which is due to dysregulation of the cell cycle. Normal cells carefully control the release of growth-promoting signals and subtly regulate the progression of the cell cycle. However, cancer cells exhibit disrupted homeostasis, resulting in malignant proliferation, which induces the loss of normal tissue architecture and function (8).

RNA metabolism (including RNA maturation, degradation and turnover and quality control), as a mediator of regulation, is required for a wide variety of biological processes. This notably includes cell proliferation, where actively proliferating cells must double their macromolecular contents and divide into two daughter cells, necessitating an increase in the biosynthesis of RNA and other molecules. However, despite their functional importance, the metabolism of RNA and the cell cycle receive less research attention than genomics and functional genomics (9). Therefore, information on the genes involved in the metabolism of RNA and the cell cycle, and specifically their involvement in HCC, is urgently required.

In the present study, DEGs were initially screened using multiple GEO microarrays and the TCGA dataset. Subsequently, enrichment analysis and protein-protein interaction (PPI) network analysis were performed. Accordingly, the amplification, increased expression and prognostic value of several central node genes were identified.

\section{Materials and methods}

Data sources. The data of the gene expression levels and DNA copy numbers between HCC and control samples were obtained from the NCBI GEO and TCGA datasets. The GEO microarray accession numbers were GSE46408, GSE50579 and GSE74656. All included datasets met the following criteria: i) They employed human liver tissue samples; ii) they contained case-control groups; and iii) they contained at least ten samples. The platform for GSE46408 was the Agilent-014850 Whole Human Genome Microarray 4x44K G4112F (Human 1A Oligo Chip V2;Welgene Biotech Co, Ltd.) and the samples comprised six pairs of HCC and their corresponding non-tumor liver parenchyma tissues. The platform for GSE50579 was the Agilent-028004 SurePrint G3 Human GE 8x60K Microarray (Agilent Technologies 2100 Bioanalyzer; Agilent Technologies Deutchland $\mathrm{GmbH}$ ) and the samples comprised seven normal liver tissues and $61 \mathrm{HCC}$ tissues. The platform for GSE74656 was the GeneChip ${ }^{\circledR}$ PrimeView $^{\mathrm{TM}}$ Human Gene Expression Array (with External spike-in RNAs), which contained five non-tumor HCC tissues. Furthermore, the data for 53 normal liver tissues and $351 \mathrm{HCC}$ tissues were obtained from TCGA for analysis. Only upregulated genes at the RNA level with a fold-change of $>2$ and $\mathrm{P}<0.05$ were considered.

Dataset analysis. Functional enrichment analysis of common DEGs was performed using the Metascape dataset (http://metascape.org/gp/index.html\#/main/step1) according to methods described previously (10). A PPI network was constructed using the Search Tool for the Retrieval of Interacting
Genes/proteins (STRING) online database (http://string-bd. org). The prognostic value of mRNA for HCC was assessed using the public online tool Kaplan-Meier Plotter (Www. kmplot.com) according to methods described previously (11). The gene mRNA heatmap and DNA amplification numbers for small nuclear ribonucleoprotein polypeptide E (SNRPE), small nuclear ribonucleoprotein polypeptide B and B1 (SNRPB), BOP1 ribosomal biogenesis factor (BOP1), nucleoporin 37 (NUP37), Rac GTPase activating protein 1 (RACGAP1), CAP-Gly domain containing linker protein 1 (CLIP1), microtubule associated protein RP/EB family member 1 (MAPRE1), kinesin family member 20A (KIF20A), kinesin family member 2A (KIF2A), minichromosome maintenance 10 replication initiation factor (MCM10), ubiquitin conjugating enzyme E2 C (UBE2C) and hyaluronan mediated motility receptor (HMMR) were obtained from cBioPortal (http://www.cbioportal.org/).

Overall survival and progression-free survival curves were plotted by the Kaplan-Meier method with Kaplan-Meier Plotter (www.kmplot.com) using a Cox regression model and compared using the log-rank test, and the median expression level was used as the cut-off to stratify the patients into high and low expression groups.

\section{Results}

DEGs in the GSE50579, GSE74656, GSE46408 and TCGA datasets. Venn diagram was used to analyze the upregulated genes between HCC and normal controls from different studies. Since somatic copy number alterations are associated with cancer and have been suggested as a specific therapeutic target (12), upregulated genes whose DNA copy numbers are increased in HCC were screened. The downregulated genes were ignored, as our group intends to conduct further studies to investigate whether targeting these upregulated genes may be applied to HCC therapy. According to the above-mentioned principles, a total of 2,553 upregulated genes were identified from GSE50579, GSE74656, GSE46408 and TCGA along with amplified genes in TCGA (Fig. 1A).

Subsequently, functional enrichment analysis of these upregulated genes was performed using Metascape. As indicated in Fig. 1B, metabolism of RNA and the cell cycle were the most commonly enriched terms. Enrichment networks were also established by representing each enriched term as a node and a neighboring node with Kappa similarities of $>0.3$. Nodes are colored to represent their cluster memberships (Fig. 1C) or statistical P-values (Fig. 1D).

Identification of key candidate genes for diagnosis of HCC. The genes enriched in the metabolism of RNA and the cell cycle were used to perform a PPI network analysis using the STRING database (Fig. 2). In addition, the central node genes were identified. For the metabolism of RNA, the genes with $>50$ connections/interactions were further studied. The 22 most connected genes were ribosomal protein S 5, RPS28, RPS19, RPS10, RPS13, RPS20, ribosomal protein L27, UPF1 RNA helicase and ATPase, ribosomal protein lateral stalk subunit P0, RPL28, cleavage and polyadenylation specific factor 1, RPL18, SNRPE, SNRPB, ubiquitin A-52 residue ribosomal protein fusion product 1 (UBA52), protein phosphatase 2 
A

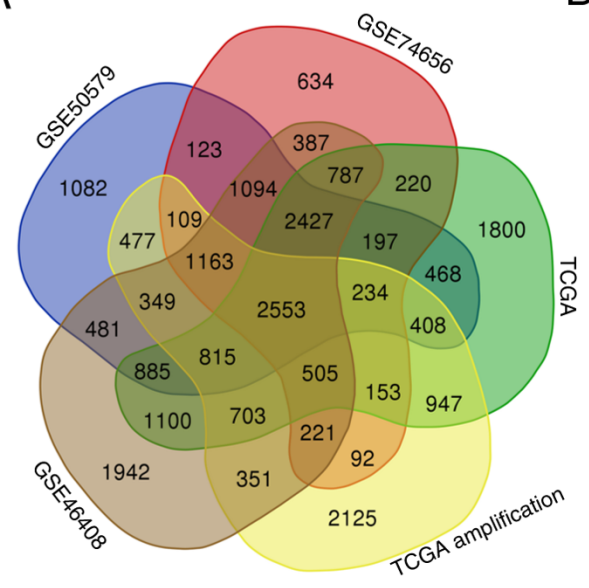

B

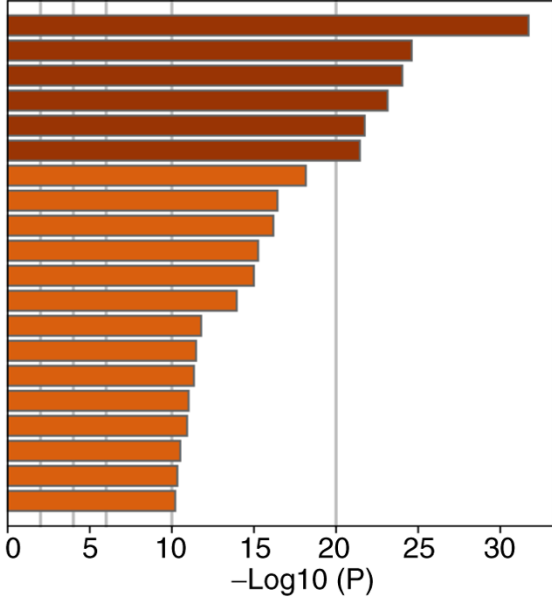

R-HSA-8953854: Metabolism of RNA R-HSA-1640170: Cell cycle R-HSA-72766: Translation R-HSA-5663205: Infectious disease R-HSA-1280218: Adaptive immune system GO:0006412: Translation

GO:0022613: Ribonucleoprotein complex biogenesis GO:0010564: Regulation of cell cycle process R-HSA-8953897: Cellular responses to external stimul GO:0006403: RNA localization

GO:0051301: Cell division

R-HSA-199991: Membrane trafficking

R-HSA-3700989: Transcriptional regulation by TP53 hsa05016: Huntington's disease

GO:0051052: Regulation of DNA metabolic process GO:0019882: Antigen processing and presentation R-HSA-3247509: Chromatin modifying enzymes

GO:0007005: Mitochondrion organization

GO:0010638: Positive regulation of organelle organization GO:0140053: Mitochondrial gene expression

\section{C}

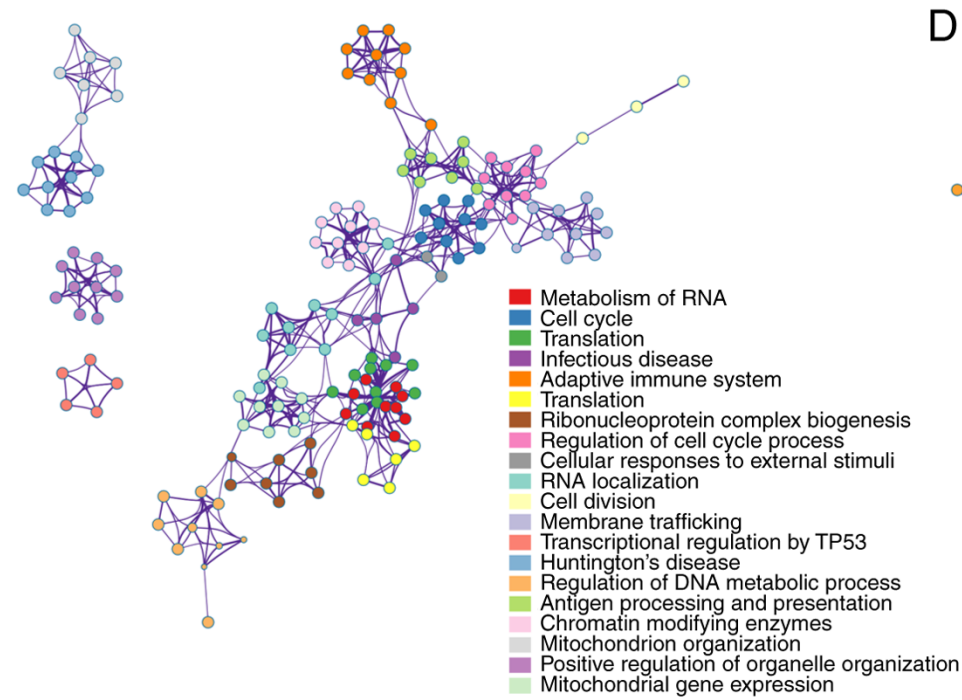

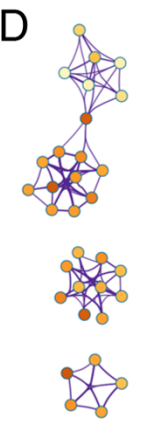

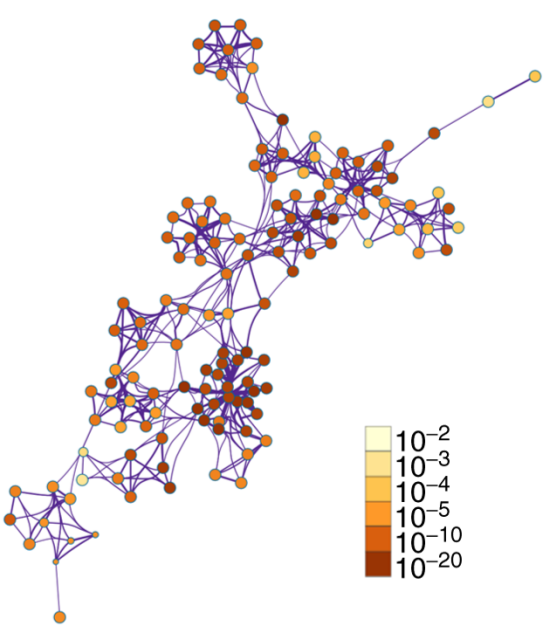

Figure 1. DEGs from the GSE50579, GSE74656, GSE46408 and TCGA datasets. (A) Venn diagram presenting the upregulated DEGs, 2,553 of which were shared between the different datasets. (B) Metascape enrichment analysis for the DEGs. (C) Enrichment networks of DEGs, represented by cluster memberships, were obtained using the Metascape dataset; (D) enrichment networks represented by statistical P-values obtained using the Metascape dataset. TCGA, The Cancer Genome Atlas; GO, gene ontology; Hsa, Homo sapiens; DEGs, differentially expressed genes.

catalytic subunit alpha (PPP2CA), RPL30, ribosomal protein L26 like 1, ribosomal protein S18, BOP1, RPL23 and RNA polymerase II, I and III subunit H (Table I). Furthermore, univariate Cox regression was performed to establish whether the above genes may serve as predictors for overall survival and progression-free survival using data from the Kaplan-Meier Plotter dataset, and sources for the database include GEO, EGA and TCGA. The median of mRNA level in the tumor samples was used as a cut-off to stratify the patients into high and low expression groups. As presented in Table I, only SNRPE, SNRPB and BOP1 were associated with the overall survival of patients with HCC. Specifically, patients with HCC and high mRNA levels for SNRPE, SNRPB or BOP1 had poorer overall survival than those with low levels of SNRPE or BOP1 (Table I; Fig. 3). However, only SNRPB appeared to be associated with progression-free survival of patients with HCC (Fig. 4).

Next, the expression profiles of SNRPE, SNRPB or BOP1 and their DNA alteration were analyzed using the cBioPortal dataset (Fig. 5). The results showed that their DNA are both amplified.
In terms of the cell cycle, the genes with $>50$ connections/interactions were further studied, and the top 23 central node genes were subjected to univariate Cox regression analysis to determine their predictive value for overall survival. These were PPP2CA, UBA52, MCM3, protein phosphatase 2 regulatory subunit B'delta, NUP37, dynein cytoplasmic 1 intermediate chain 2, RACGAP1, H2B clustered histone 15, cell division cycle 27, CLIP1, H2B clustered histone 3, exportin 1, actin gamma 1, histone cluster 1, H2bm, MAPRE1, proteasome 20S subunit beta 3, KIF20A, KIF2A, MCM10, DSN1 component of MIS12 kinetochore complex, UBE2C and HMMR (Table II). The analysis indicated that NUP37, RACGAP1, CLIP1, MAPRE1, KIF20A, KIF2A, MCM10, UBE2C and HMMR might serve as the prognostic markers for overall survival (Table II; Fig. 3). While, NUP37, RACGAP1, KIF20A, MCM10, UBE2C and HMMR also were closely correlated with progression-free survival for patients with $\mathrm{HCC}$, suggesting these genes might also serve as prognostic markers for progression-free survival for patients with HCC (Fig. 4). As presented in Fig. 5, the expression 
A

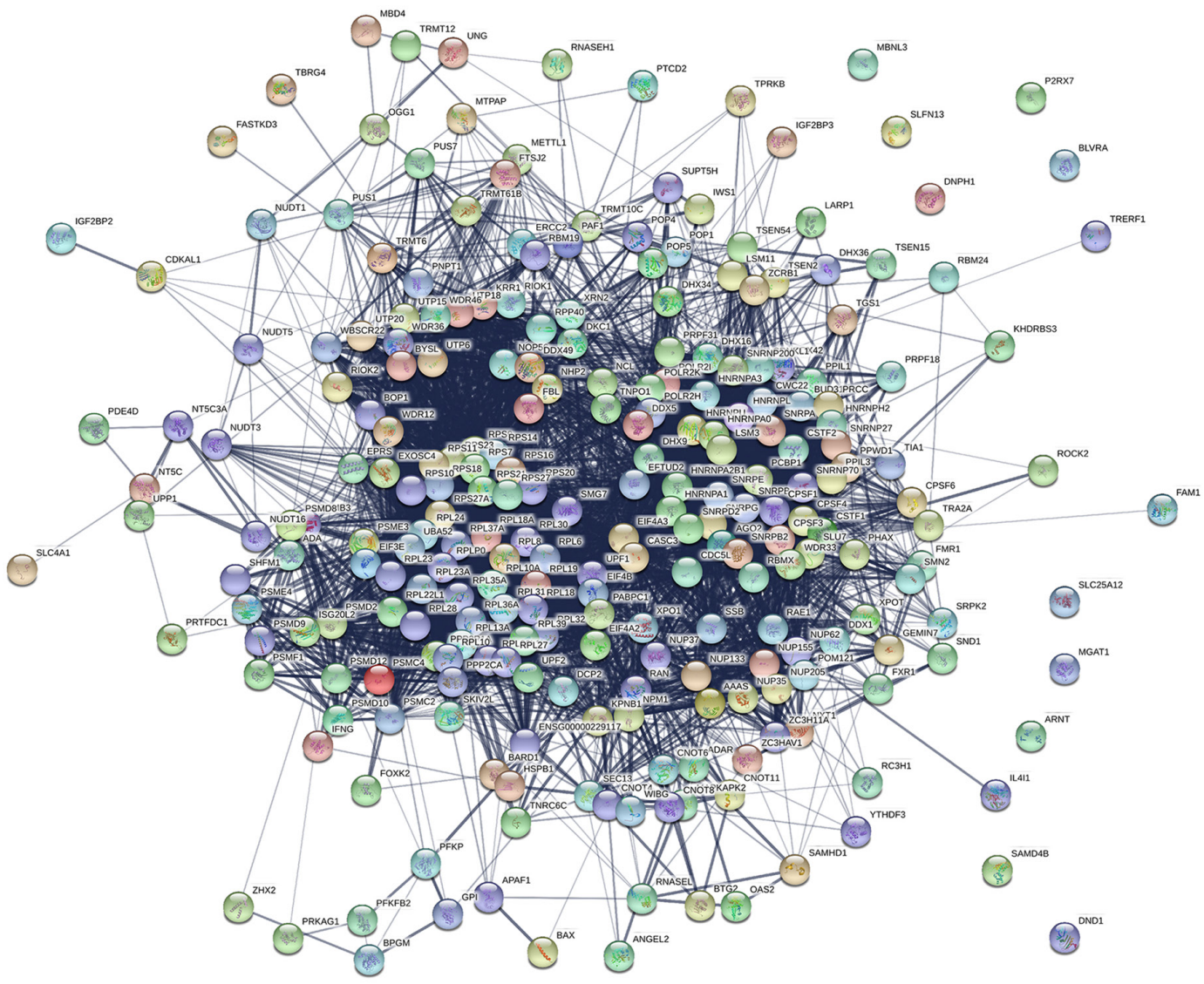

B

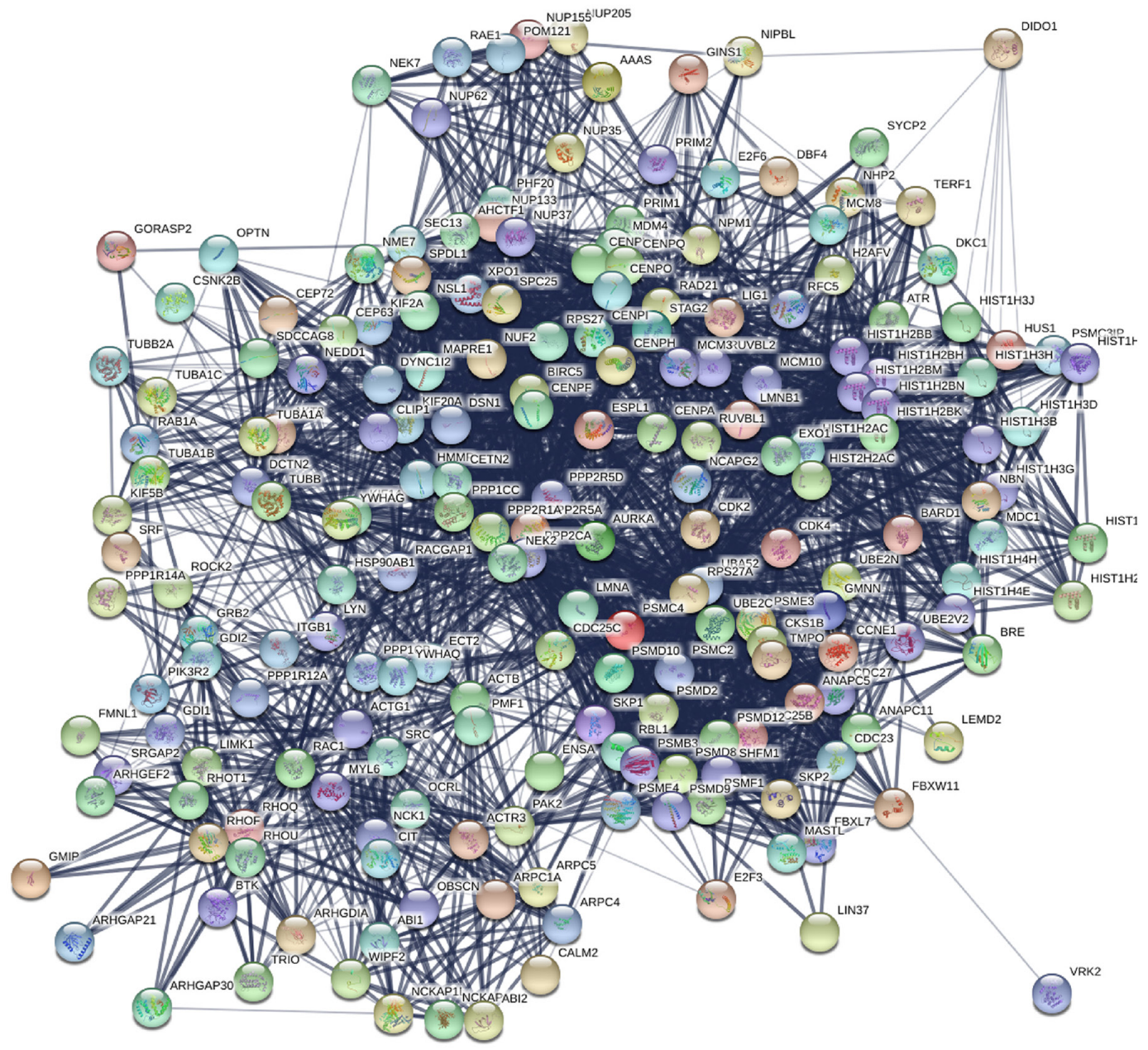

Figure 2. PPI networks according to the STRING online database. (A) PPI network for genes involved in the metabolism of RNA; (B) PPI network for genes involved in the cell cycle. PPI, protein-protein interaction. 
Table I. Prognostic value of mRNA levels of the key genes from PPI (enriched in metabolism of RNA) for overall survival of patients with hepatocellular carcinoma using the Kaplan-Meier Plotter dataset.

\begin{tabular}{|c|c|c|c|}
\hline Factor & Numbers of international proteins & HR $(95 \% \mathrm{CI})$ & $P$ value \\
\hline RPS5 & 77 & $1.28(0.89-1.83)$ & 0.18 \\
\hline RPS28 & 77 & $0.95(0.66-1.36)$ & 0.77 \\
\hline RPS19 & 76 & $1.02(0.71-1.46)$ & 0.91 \\
\hline RPS10 & 76 & $1.01(0.71-1.44)$ & 0.96 \\
\hline RPS13 & 67 & $1.30(0.91-1.86)$ & 0.15 \\
\hline RPS20 & 65 & $1.12(0.78-1.60)$ & 0.54 \\
\hline RPL27 & 60 & $1.20(0.84-1.72)$ & 0.31 \\
\hline UPF1 & 59 & $0.80(0.56-1.14)$ & 0.21 \\
\hline RPLP0 & 58 & $1.14(0.79-1.62)$ & 0.49 \\
\hline RPL28 & 58 & $0.88(0.61-1.25)$ & 0.47 \\
\hline CPSF1 & 58 & $1.26(0.88-1.80)$ & 0.21 \\
\hline RPL18 & 57 & $1.08(0.76-1.54)$ & 0.67 \\
\hline SNRPE & 54 & $1.56(1.09-2.23)$ & 0.015 \\
\hline SNRPB & 54 & $1.47(1.02-2.11)$ & 0.036 \\
\hline UBA52 & 53 & $1.05(0.74-1.50)$ & 0.78 \\
\hline PPP2CA & 53 & $0.99(0.69-1.41)$ & 0.94 \\
\hline RPL30 & 52 & $0.89(0.62-1.27)$ & 0.53 \\
\hline RPL26L1 & 52 & $1.12(0.78-1.60)$ & 0.53 \\
\hline RPS18 & 52 & $1.08(0.75-1.54)$ & 0.68 \\
\hline BOP1 & 52 & $1.72(1.20-2.48)$ & 0.0030 \\
\hline RPL23 & 51 & $0.84(0.59-1.20)$ & 0.34 \\
\hline POLR2H & 51 & $1.41(0.98-2.02)$ & 0.06 \\
\hline
\end{tabular}

HR, hazard ratio. Numbers of international proteins, the numbers of proteins that are associated with the indicated protein from the PPI; PPI, protein-protein interactions; SNRP, small nuclear ribonucleoprotein polypeptide; BOP1, BOP1 ribosomal biogenesis factor; RPS ribosomal protein; UPF1, UPF1 RNA helicase and ATPase; RPL, ribosomal protein lateral stalk subunit; CPSF1, cleavage and polyadenylation specific factor 1; UBA52, ubiquitin A-52 residue ribosomal protein fusion product 1; PPP2CA, protein phosphatase 2 catalytic subunit $\alpha$; RPL26L1, ribosomal protein L26 like 1; RPS18, ribosomal protein S18; POLR2H, RNA polymerase II, I and III subunit H.

profile of MCM3, RACGAP1, MAPRE1, KIF20A, KIF2A, MCM10 and HMMR and DNA alteration of these genes were analyzed using the cBioPortal dataset. The results showed that their DNA are all amplified.

\section{Discussion}

The tumorigenesis and progression of HCC involve a multistep process during which cells undergo complex changes, including accumulating mutations, which lead to activation of oncogenes and loss of tumor suppressor genes. These genes are implicated in multiple pathways that may regulate different steps of carcinogenesis. For instance, certain steps are essential for driving cell transformation, while others have indispensable roles in cancer progression or in the acquisition of characteristics required for metastasis (13). Therefore, identifying such genes is crucial for cancer therapy. However, HCC cells are morphologically and genetically heterogeneous. This heterogeneity partly accounts for the complexity of HCC (14). Therefore, in view of this complexity, it is important to integrate data from different independent studies in order to perform robust analyses.

Accordingly, in the present study, DEGs were identified from GEO and TCGA datasets. Functional enrichment analysis revealed that these genes were mainly associated with RNA metabolism and the cell cycle.

A portion of the genome is known to be transcribed into RNAs, whose biological functions are still being determined. In view of the importance of RNAs, there is a significant turnover of RNAs associated with cell maintenance, repair and modulation, even in quiescent cells. Proliferating cells must upregulate the biosynthesis of RNA and DNA to support cell division $(9,15-18)$. Sustained chronic proliferation is the most fundamental characteristic of cancer cells (8). The proliferation of cancer cells necessitates the upregulation of RNA biosynthesis. In line with this, the present study demonstrated that the DEGs were mainly involved in RNA metabolism. Furthermore, it is widely accepted that the sustained proliferation of cancer cells is realized through deregulation of the cell cycle (19). Accordingly, in the present study, it was indicated that the upregulated genes were also implicated in the cell cycle.

PPI network analysis offers more detailed information on the connections among the upregulated genes in HCC samples. Three genes associated with the overall survival of HCC patients, i.e., SNRPE, SNRPB and BOP1, which are involved in the metabolism of RNA, were screened. 

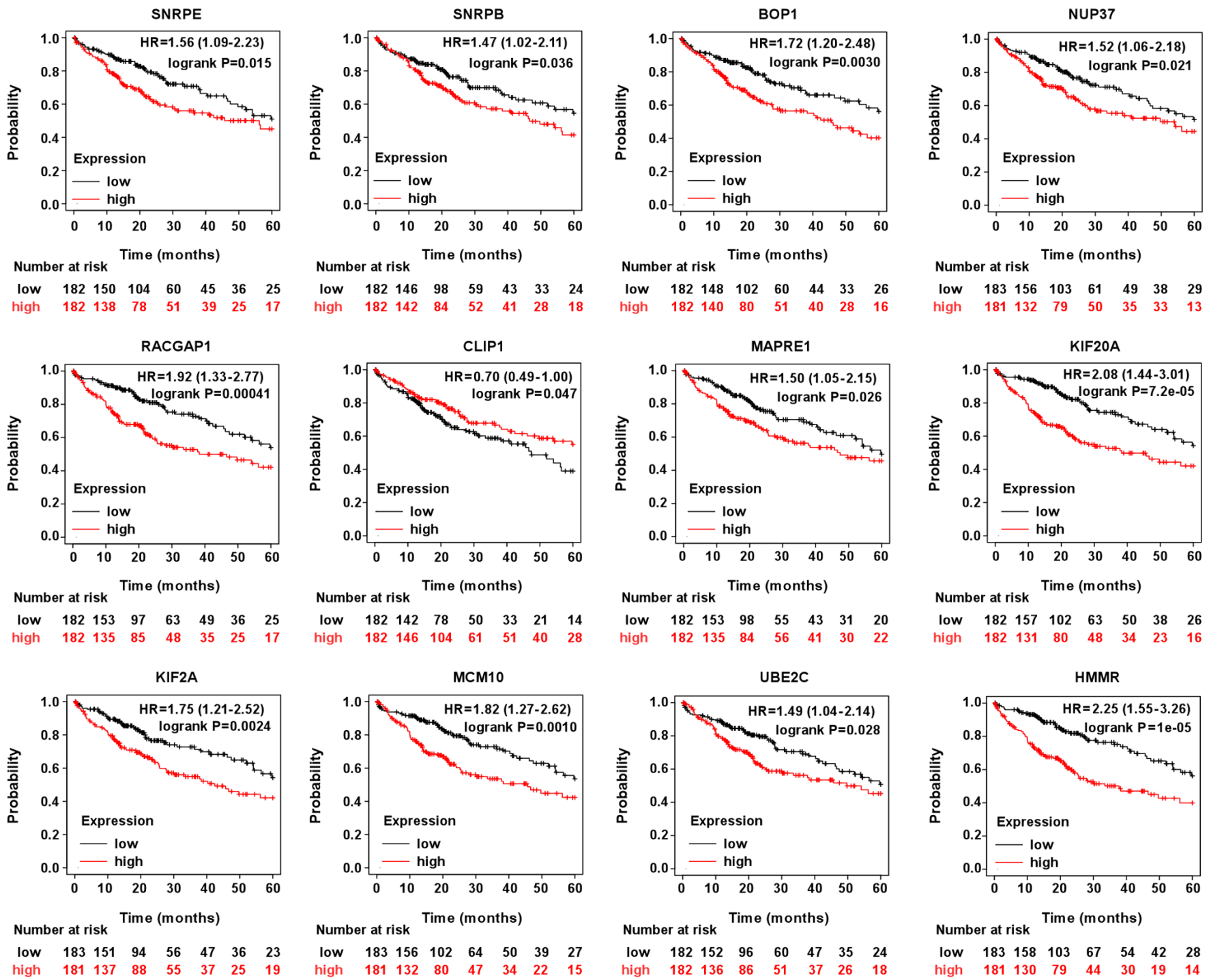

Figure 3. Prognostic value of the indicated mRNA levels on the overall survival of patients with hepatocellular carcinoma. HR, hazard ratio (provided with 95\% CI). SNRPE, small nuclear ribonucleoprotein polypeptide E; BOP1, BOP1 ribosomal biogenesis factor; RACGAP1, Rac GTPase activating protein 1; MAPRE1, microtubule associated protein RP/EB family member 1; KIF; kinesin family member; MCM10, minichromosome maintenance 10 replication initiation factor; HMMR, hyaluronan mediated motility receptor; UBE2C, ubiquitin conjugating enzyme E2 C; CLIP1, CAP-Gly domain containing linker protein 1; SNRPB, small nuclear ribonucleoprotein polypeptide B and B1; NUP37; nucleoporin 37.

SNRPE and SNRPB are a central component of U small nuclear ribonucleoproteins, which are the main components of pre-mRNA processing spliceosomes. It has been reported that SNRPE promotes the proliferation of HCC cells (20). BOP1 is a nucleolar protein that is involved in ribosomal RNA processing and ribosome assembly. It has been reported that it promotes epithelial-to-mesenchymal transition (21).

In a similar manner, seven genes, i.e., NUP37, RACGAP1, CLIP1, MAPRE1, KIF20A, KIF2A, MCM10, UBE2C and HMMR, which regulate the cell cycle in HCC, were screened. Their high expression indicated poor prognosis for patients with HCC. MCM10 belongs to the MCM protein, which is involved in the initiation of eukaryotic genome replication. Their deregulation is observed in multiple cancer types, including prostate cancer (22), HCC (23-25) and renal cell carcinoma (26). RACGAP1 is a GTPase-activating protein that is a component of the central spindlin complex. It is able to bind to activated forms of Rho GTPases and stimulates
GTP hydrolysis to induce negative regulation of Rho-mediated signals. It has been reported that high RACGAP1 is correlated with a high rate of post-resection recurrent HCC and may be used as a potential molecular target in the design of therapeutic methods for HCC (27). Furthermore, RACGAP1 participates in the progression of multiple cancer types (28-31).

MAPRE1 belongs to the RP/EB family and was organically identified by its binding with the APC protein. It is involved in the modulation of microtubule structures and chromosome stability. Its deregulation is associated with various cancer types, including colorectal cancer (32), gastric cancer (33), acute lymphoblastic leukemia (34) and HCC (35).

KIF2A and KIF20A belong to the kinesin family, which is a plus end-directed motor required for normal mitotic progression. Their deregulation is implicated in multiple cancer types (36-40).

HMMR forms a complex with BRCA1 and BRCA2 to regulate cell motility. It has been documented that HMMR 


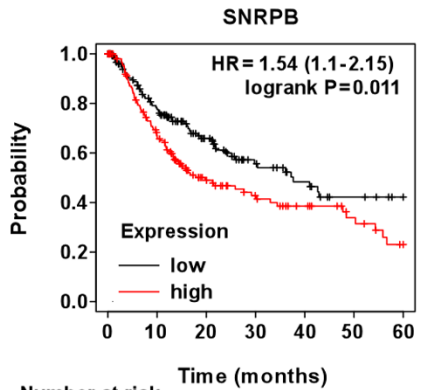

Number at risk Time (months)

$\begin{array}{llllllll}\text { low } & 158 & 102 & 60 & 35 & 25 & 17 & 13\end{array}$ $\begin{array}{llllllll}\text { high } & 158 & 92 & 45 & 31 & 22 & 14 & 7\end{array}$

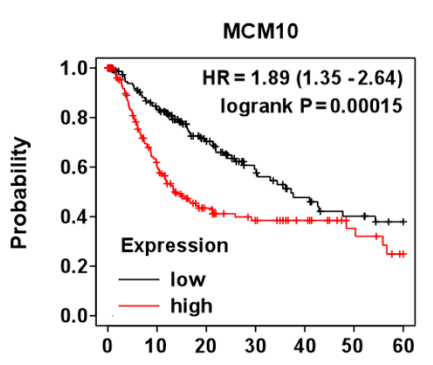

Number at risk Time (months)

$\begin{array}{llllllll}\text { low } & 159 & 115 & 66 & 39 & 27 & 20 & 15\end{array}$

high $\quad \begin{array}{lllllll}157 & 79 & 39 & 27 & 20 & 11 & 5\end{array}$

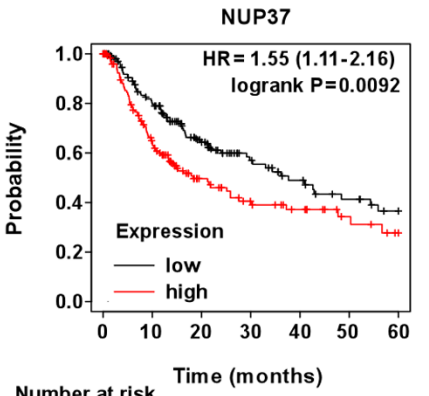

$\begin{array}{llllllll}\text { low } & 158 & 110 & 64 & 39 & 28 & 20 & 14\end{array}$

$\begin{array}{llllllll}\text { high } & 158 & 84 & 41 & 27 & 19 & 11 & 6\end{array}$

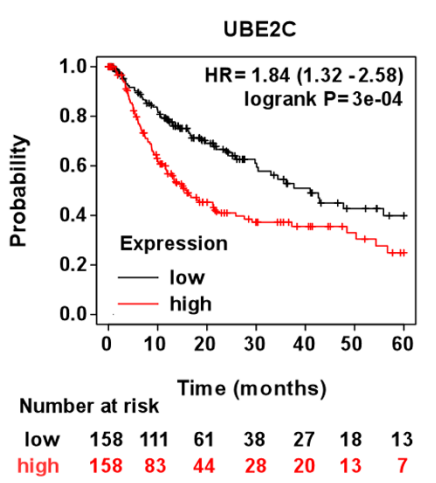

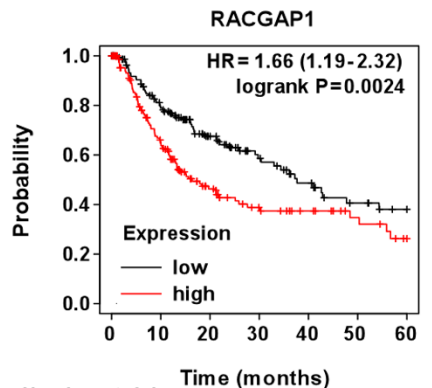

Number at risk Time (months)

$\begin{array}{llllllll}\text { low } & 159 & 108 & 63 & 39 & 27 & 18 & 13\end{array}$ $\begin{array}{llllllll}\text { high } & 157 & 86 & 42 & 27 & 20 & 13 & 7\end{array}$

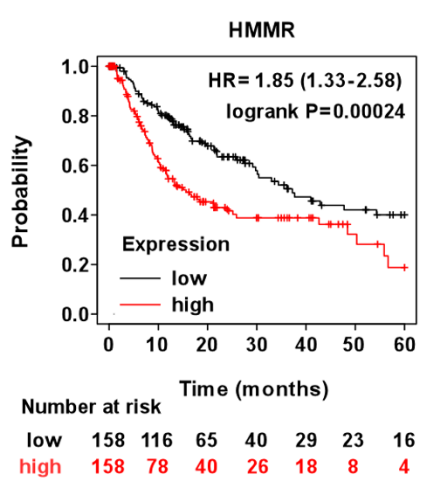

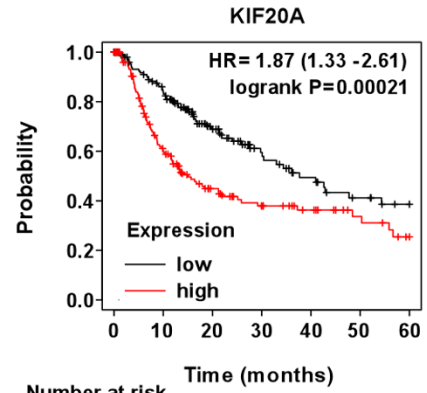

$\begin{array}{llllllll}\text { low } & 158 & 115 & 62 & 37 & 26 & 18 & 13\end{array}$ $\begin{array}{llllllll}\text { high } & 158 & 79 & 43 & 29 & 21 & 13 & 7\end{array}$

Figure 4. Prognostic value of the indicated mRNA levels on the progression-free survival of patients with hepatocellular carcinoma. Genes significantly associated with progression-free survival were selected for analysis. HR, hazard ratio (provided with 95\% CI). RACGAP1, Rac GTPase activating protein 1; KIF; kinesin family member; MCM10, minichromosome maintenance 10 replication initiation factor; HMMR, hyaluronan mediated motility receptor; UBE2C, ubiquitin conjugating enzyme E2 C; NUP37; nucleoporin 37; SNRPB, small nuclear ribonucleoprotein polypeptide B and B1.

\begin{abstract}
SNRPE 20\%

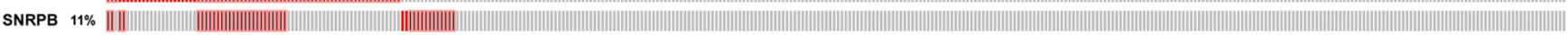
BOP1 35\% NUP37 6\% RACGAP1 6\%

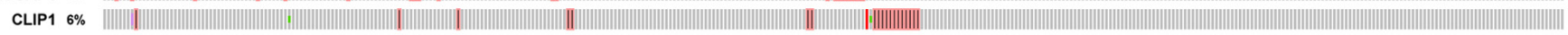
MAPre1 $8 \%$.

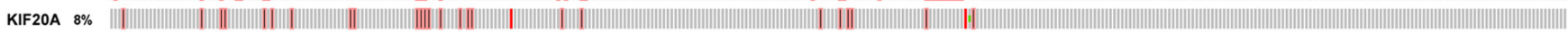

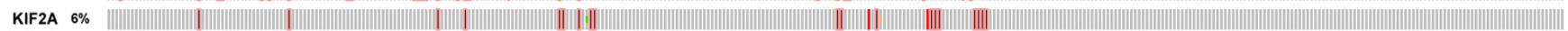
мсм10 10\% UBE2C HMme 9\%
\end{abstract}
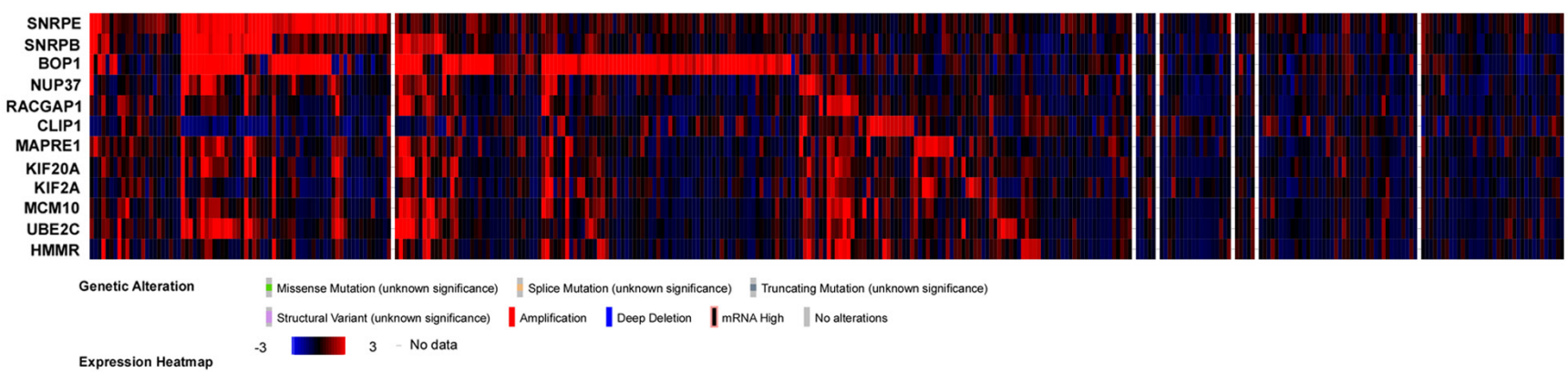

Figure 5. DNA alteration (upper panel) and mRNA profile (lower panel) data for the genes implicated in hepatocellular carcinoma. SNRPE, small nuclear ribonucleoprotein polypeptide E; SNRPB, small nuclear ribonucleoprotein polypeptide B and B1; BOP1, BOP1 ribosomal biogenesis factor; RACGAP1, Rac GTPase activating protein 1; MAPRE1, microtubule associated protein RP/EB family member 1; KIF; kinesin family member; MCM10, minichromosome maintenance 1 replication initiation factor; HMMR, hyaluronan mediated motility receptor; UBE2C, ubiquitin conjugating enzyme E2 C; NUP37; nucleoporin 37.

maintains the stemness and tumorigenicity of glioblastoma stem-like cells (41). Another study has indicated that HMMR may be used as a biomarker for neutropenia induced by chemotherapy in patients with breast cancer (42).
All of these genes are highly interacting/connected genes, suggesting that they may have important roles in HCC. Since they regulate RNA metabolism and cell cycle, targeting them as a cancer therapy would not be specific and would, 
Table II. Prognostic value of mRNA levels of the key genes from the PPI (enriched in cell cycle) for overall survival of patients with hepatocellular carcinoma using the Kaplan-Meier Plotter dataset.

\begin{tabular}{lccc}
\hline Factor & Numbers of international proteins & HR $(95 \% \mathrm{CI})$ & P-value \\
\hline PPP2CA & 60 & $0.99(0.69-1.41)$ & 0.94 \\
UBA52 & 56 & $1.05(0.74-1.50)$ & 0.78 \\
MCM3 & 46 & $1.58(1.10-2.26)$ & 0.013 \\
PPP2R5D & 45 & $1.10(0.77-1.57)$ & 0.60 \\
NUP37 & 43 & $1.52(1.06-2.18)$ & 0.021 \\
DYNC1I2 & 43 & $1.12(0.78-1.60)$ & 0.54 \\
RACGAP1 & 41 & $1.92(1.33-2.77)$ & 0.00041 \\
HIST1H2BN & 39 & $1.08(0.76-1.54)$ & 0.67 \\
CDC27 & 38 & $1.25(0.88-1.80)$ & 0.22 \\
CLIP1 & 38 & $0.70(0.49-1.00)$ & 0.047 \\
HIST1H2BB & 38 & $1.26(0.77-2.06)$ & 0.35 \\
XPO1 & 38 & $1.29(0.90-1.85)$ & 0.16 \\
HIST1H2BH & $1.40(0.97-2.00)$ & 0.068 \\
ACTG1 & 37 & $1.40(0.97-2.01)$ & 0.067 \\
HIST1H2BM & 37 & $0.86(0.53-1.40)$ & 0.55 \\
MAPRE1 & 36 & $1.50(1.05-2.15)$ & 0.026 \\
PSMB3 & 35 & $0.84(0.59-1.21)$ & 0.35 \\
KIF20A & 34 & $2.08(1.44-3.01)$ & $7.20 \times 10^{-5}$ \\
KIF2A & 34 & $1.75(1.21-2.52)$ & 0.0024 \\
MCM10 & 34 & $1.82(1.27-2.62)$ & 0.0010 \\
DSN1 & 33 & $1.20(0.84-1.71)$ & 0.32 \\
UBE2C & 32 & $1.49(1.04-2.14)$ & 0.028 \\
HMMR & 32 & $2.25(1.55-3.26)$ & $1.00 \times 10^{-5}$ \\
\hline
\end{tabular}

HR, hazard ratio; numbers of international proteins, the numbers of proteins that are associated with the indicated protein from the PPI; PPI, protein-protein interactions. PPP2CA, protein phosphatase 2 catalytic subunit $\alpha$; UBA52, ubiquitin A-52 residue ribosomal protein fusion product 1; MCM3, minichromosome maintenance complex; PPP2R5D, protein phosphatase 2 regulatory subunit B'delta; NUP37, nucleoporin 37; DYNC1I2, dynein cytoplasmic 1 intermediate chain 2; RACGAP1, Rac GTPase activating protein; HIST1H2BN, H2B clustered histone 15; CDC27, cell division cycle 27; CLIP1, CAP-Gly domain containing linker protein 1; HIST1H2BB, H2B clustered histone 3; XPO1, exportin 1; ACTG1, actin gamma 1; HIST1H2BM, histone cluster 1, H2bm; PSMB3, MAPRE1, proteasome 20S subunit beta 3; KIF, kinesin family member; DSN1, DSN1 component of MIS12 kinetochore complex; UBE2C, ubiquitin conjugating enzyme E2 C; HMMR, hyaluronan mediated motility receptor.

therefore, be expected to have severe side effects. However, they are significantly associated with overall survival for patients with HCC; therefore, they may serve as prognosis markers for patients with HCC. As another limitation, only a univariate analysis was performed to determine the association of these genes with survival, while multivariate analysis of single genes, or of a combined gene signature, may have provided an independent prognostic marker, which might provide more valuable for prognosis of $\mathrm{HCC}$, and should be provided in a future study.

\section{Acknowledgements}

Not applicable.

\section{Funding}

This work was supported by grants from the Science and Technology Projects Foundation of Guangzhou City (grant nos. 201804010416 and 201904010355 ) and the Guangzhou Zengcheng District Science and Technology Innovation Project (grant no. 2021049).

\section{Availability of data and materials}

The datasets used and/or analyzed during the present study are available from the corresponding author on reasonable request.

\section{Authors' contributions}

XHL and ZPF designed the study. FQL, RY, LYY, HS and HLL completed the data acquisition and analysis. ZPF wrote the manuscript. All authors have read and approved the final version of the manuscript. ZPF, XHL, FQL, RY, LYY, HS and HLL confirm the authenticity of all the raw data. 


\section{Ethics approval and consent to participate}

Not applicable.

\section{Patient consent for publication}

Not applicable.

\section{Competing interests}

The authors declare that they have no competing interests.

\section{References}

1. Bray F, Ferlay J, Soerjomataram I, Siegel RL, Torre LA and Jemal A: Global cancer statistics 2018: GLOBOCAN estimates of incidence and mortality worldwide for 36 cancers in 185 countries. CA Cancer J Clin 68: 394-424, 2018.

2. Chen W, Zheng R, Baade PD, Zhang S, Zeng H, Bray F, Jemal $\mathrm{A}$, Yu XQ and He J: Cancer statistics in China, 2015. CA Cancer J Clin 66: 115-132, 2016.

3. Han D, Li J, Wang H, Su X, Hou J, Gu Y, Qian C, Lin Y, Liu X, Huang M, et al: Circular RNA circMTO1 acts as the sponge of microRNA-9 to suppress hepatocellular carcinoma progression. Hepatology 66: 1151-1164, 2017.

4. Barrett T and Edgar R: Mining microarray data at NCBI's Gene Expression Omnibus (GEO)*. Methods Mol Biol 338: 175-190, 2006

5. Clough E and Barrett T: The Gene Expression Omnibus Database. Methods Mol Biol 1418: 93-110, 2016.

6. Edgar R, Domrachev M and Lash AE: Gene Expression Omnibus: NCBI gene expression and hybridization array data repository. Nucleic Acids Res 30: 207-210, 2002.

7. Wei L, Jin Z, Yang S, Xu Y, Zhu Y and Ji Y: TCGA-assembler 2: Software pipeline for retrieval and processing of TCGA/CPTAC data. Bioinformatics 34: 1615-1617, 2018.

8. Hanahan D and Weinberg RA: Hallmarks of cancer: The next generation. Cell 144: 646-674, 2011.

9. Lane AN and Fan TW: Regulation of mammalian nucleotide metabolism and biosynthesis. Nucleic Acids Res 43: 2466-2485, 2015

10. Zhou Y, Zhou B, Pache L, Chang M, Khodabakhshi AH, Tanaseichuk O, Benner C and Chanda SK: Metascape provides a biologist-oriented resource for the analysis of systems-level datasets. Nat Commun 10: 1523, 2019.

11. Győrffy B, Surowiak P, Budczies J and Lánczky A: Online survival analysis software to assess the prognostic value of biomarkers using transcriptomic data in non-small-cell lung cancer. PLoS One 8: e82241, 2013.

12. Zhou CC, Yang F, Yuan SX, Ma JZ, Liu F, Yuan JH, Bi FR, Lin KY, Yin JH, Cao GW, et al: Systemic genome screening identifies the outcome associated focal loss of long noncoding RNA PRAL in hepatocellular carcinoma. Hepatology 63 850-863, 2016.

13. Imbeaud S, Ladeiro Y and Zucman-Rossi J: Identification of novel oncogenes and tumor suppressors in hepatocellular carcinoma. Semin Liver Dis 30: 75-86, 2010.

14. Spangenberg HC, Thimme R and Blum HE: Targeted therapy for hepatocellular carcinoma. Nat Rev Gastroentero Hepatol 6: 423-432, 2009

15. Hangauer MJ, Vaughn IW and McManus MT: Pervasive transcription of the human genome produces thousands of previously unidentified long intergenic noncoding RNAs PLoS Genet 9: e1003569, 2013.

16. Djebali S, Davis CA, Merkel A, Dobin A, Lassmann T, Mortazavi A, Tanzer A, Lagarde J, Lin W, Schlesinger F, et al Landscape of transcription in human cells. Nature 489: 101-108, 2012

17. Consortium EP; ENCODE Project Consortium: An integrated encyclopedia of DNA elements in the human genome. Nature 489: 57-74, 2012.

18. Sigoillot FD, Berkowski JA, Sigoillot SM, Kotsis DH and Guy HI: Cell cycle-dependent regulation of pyrimidine biosynthesis. J Biol Chem 278: 3403-3409, 2003.
19. Urrego D, Tomczak AP, Zahed F, Stühmer W and Pardo LA: Potassium channels in cell cycle and cell proliferation. Philos Trans R Soc Lond B Biol Sci 369: 20130094, 2014.

20. Jia D, Wei L, Guo W, Zha R, Bao M, Chen Z, Zhao Y, Ge C, Zhao F, Chen T, et al: Genome-wide copy number analyses identified novel cancer genes in hepatocellular carcinoma. Hepatology 54: 1227-1236, 2011.

21. Chung KY, Cheng IK, Ching AK, Chu JH, Lai PB and Wong $\mathrm{N}$ : Block of proliferation 1 (BOP1) plays an oncogenic role in hepatocellular carcinoma by promoting epithelial-to-mesenchymal transition. Hepatology 54: 307-318, 2011.

22. Cui F, Hu J, Ning S, Tan J and Tang H: Overexpression of MCM10 promotes cell proliferation and predicts poor prognosis in prostate cancer. Prostate 78: 1299-1310, 2018.

23. Liu Z, Li J, Chen J, Shan Q, Dai H, Xie H, Zhou L, Xu X and Zheng S: MCM family in HCC: MCM6 indicates adverse tumor features and poor outcomes and promotes S/G2 cell cycle progression. BMC Cancer 18: 200, 2018.

24. Zhuang L, Yang Z and Meng Z: Upregulation of BUB1B, CCNB1, CDC7, CDC20, and MCM3 in tumor tissues predicted worse overall survival and disease-free survival in hepatocellular carcinoma patients. BioMed Res Int 2018: 7897346, 2018.

25. Yang Q, Xie B, Tang H, Meng W, Jia C, Zhang X, Zhang Y, Zhang J, Li H and Fu B: Minichromosome maintenance 3 promotes hepatocellular carcinoma radioresistance by activating the NF- $\kappa$ B pathway. J Exp Clin Cancer Res 38: 263 , 2019.

26. Gao Z, Man X, Li Z, Bi J, Liu X, Li Z, Li J, Zhang Z and Kong C: PLK1 promotes proliferation and suppresses apoptosis of renal cell carcinoma cells by phosphorylating MCM3. Cancer Gene Ther 27: 412-423, 2020.

27. Wang SM, Ooi LL and Hui KM: Upregulation of Rac GTPase-activating protein 1 is significantly associated with the early recurrence of human hepatocellular carcinoma. Clin Cancer Res 17: 6040-6051, 2011.

28. Wang C, Wang W, Liu Y, Yong M, Yang Y and Zhou H: Rac GTPase activating protein 1 promotes oncogenic progression of epithelial ovarian cancer. Cancer Sci 109: 84-93, 2018.

29. Mi S, Lin M, Brouwer-Visser J, Heim J, Smotkin D, Hebert T, Gunter MJ, Goldberg GL, Zheng D and Huang GS: RNA-seq Identification of RACGAP1 as a Metastatic Driver in Uterine Carcinosarcoma. Clin Cancer Res 22: 4676-4686, 2016.

30. Imaoka H, Toiyama Y, Saigusa S, Kawamura M, Kawamoto A, Okugawa Y, Hiro J, Tanaka K, Inoue Y, Mohri Y, et al: RacGAP1 expression, increasing tumor malignant potential, as a predictive biomarker for lymph node metastasis and poor prognosis in colorectal cancer. Carcinogenesis 36: 346-354, 2015.

31. Saigusa S, Tanaka K, Mohri Y, Ohi M, Shimura T, Kitajima T, Kondo S, Okugawa Y, Toiyama Y, Inoue Y, et al: Clinical significance of RacGAP1 expression at the invasive front of gastric cancer. Gastric Cancer 18: 84-92, 2015.

32. Taguchi A, Rho JH, Yan Q, Zhang Y, Zhao Y, Xu H, Tripathi SC, Wang H, Brenner DE, Kucherlapati M, et al: MAPRE1 as a plasma biomarker for early-stage colorectal cancer and adenomas. Cancer Prev Res (Phila) 8: 1112-1119, 2015.

33. Kim K, Lee HC, Park JL, Kim M, Kim SY, Noh SM, Song KS, Kim JC and Kim YS: Epigenetic regulation of microRNA-10b and targeting of oncogenic MAPRE1 in gastric cancer. Epigenetics 6: 740-751, 2011.

34. Fu JF, Hsu HC and Shih LY: MLL is fused to EB1 (MAPRE1), which encodes a microtubule-associated protein, in a patient with acute lymphoblastic leukemia. Genes Chromosomes Cancer 43: 206-210, 2005.

35. Chen RX, Song HY, Dong YY, Hu C, Zheng QD, Xue TC, Liu XH, Zhang Y, Chen J, Ren ZG, et al: Dynamic expression patterns of differential proteins during early invasion of hepatocellular carcinoma. PLoS One 9: e88543, 2014.

36. Xie T, Li X, Ye F, Lu C, Huang H, Wang F, Cao X and Zhong C: High KIF2A expression promotes proliferation, migration and predicts poor prognosis in lung adenocarcinoma. Biochem Biophys Res Commun 497: 65-72, 2018.

37. Zhang X, Ma C, Wang Q, Liu J, Tian M, Yuan Y, Li X and $\mathrm{Qu} X$ : Role of KIF2A in the progression and metastasis of human glioma. Mol Med Rep 13: 1781-1787, 2016.

38. Wang J, Ma S, Ma R, Qu X, Liu W, Lv C, Zhao S and Gong Y: KIF2A silencing inhibits the proliferation and migration of breast cancer cells and correlates with unfavorable prognosis in breast cancer. BMC Cancer 14: 461, 2014. 
39. Duan J, Huang W and Shi H: Positive expression of KIF20A indicates poor prognosis of glioma patients. OncoTargets Ther 9: 6741-6749, 2016.

40. Stangel D, Erkan M, Buchholz M, Gress T, Michalski C, Raulefs S, Friess H and Kleeff J: Kif20a inhibition reduces migration and invasion of pancreatic cancer cells. J Surg Res 197: 91-100, 2015.

41. Tilghman J, Wu H, Sang Y, Shi X, Guerrero-Cazares H, Quinones-Hinojosa A, Eberhart CG, Laterra J and Ying M: HMMR maintains the stemness and tumorigenicity of glioblastoma stem-like cells. Cancer Res 74: 3168-3179, 2014.
42. Bidadi B, Liu D, Kalari KR, Rubner M, Hein A, Beckmann MW Rack B, Janni W, Fasching PA, Weinshilboum RM, et al: Pathway-Based Analysis of Genome-Wide Association Data Identified SNPs in HMMR as Biomarker for ChemotherapyInduced Neutropenia in Breast Cancer Patients. Front Pharmacol 9: 158, 2018.

(c) (1) () This work is licensed under a Creative Commons Attribution-NonCommercial-NoDerivatives 4.0 International (CC BY-NC-ND 4.0) License. 